\title{
Visión sistémica del surgimiento de las Radios Populares, Alternativas y Comunitarias en el estado Zulia*
}

\author{
Useche, María Cristina** \\ Artigas, Wileidys ${ }^{* * *}$
}

\section{Resumen}

El objetivo del trabajo se centró en establecer los subsistemas de las radios populares, alternativas y comunitarias (PAC) en el estado Zulia. El abordaje del evento fue desde la teoría de sistemas de la administración, haciendo una descripción de los elementos que conforman cada subsistema. El tipo de investigación es descriptiva-transeccional. La población estuvo conformada por 25 radios PAC habilitadas por la Comisión Nacional de Telecomunicaciones en la región Zulia, aplicándose un censo poblacional. La técnica de recolección de datos fue la entrevista, mediante un cuestionario semi-estructurado. Entre los resultados se evidencian elementos, como: la ínter conectividad técnico, político y social, así como sentido de pertenencia e identidad en el trabajo comunicacional. Se concluye que el sistema bajo el cual se desenvuelven las radios está conformada por cinco subsistemas, a saber: objetivos y valores, estructura, psicosocial, técnico y administrativo; la integración de los mismos conforman el sistema.

Palabras clave: Radios Populares, Alternativas y Comunitarias, subsistemas, estructura, relaciones.

\section{Recibido: 04-10-10. Aceptado: 21-03-11}

* $\quad$ Resultados de proyecto de investigación, titulado: "Estrategias para la Autosostenibilidad de Las Radios Populares, Alternativas y Comunitarias en el municipio Maracaibo financiado por el Consejo de Desarrollo Científico y Humanístico (CONDES) No. CH-0426-10.

** Investigadora del Centro de Estudios de la Empresa de la Facultad de Ciencias Económicas y Sociales de la Universidad del Zulia, en la línea de investigación de estrategias de modernización empresarial. Miembro del Comité Académico del Doctorado en Ciencias Sociales. Mención Gerencia de la Universidad el Zulia. Docente de la Universidad del Zulia. Economista. Magíster en Gerencia de Empresas, Mención Mercadeo. Doctora en Ciencias Económicas. E-mail: mariauseche@yahoo.es. Código Postal 4002.

*** Editora de la Revista TELOS y Miembro del Comité Académico de la Maestría en Gerencia Empresarial de la Universidad Rafael Belloso Chacín. Investigadora del Centro de Estudios de la Empresa de la Facultad de Ciencias Económicas y Sociales de la Universidad del Zulia, en la línea de investigación de estrategias de modernización empresarial. Licenciada en Administración. Magíster en Gerencia de Empresas, Mención Operaciones. Doctora en Ciencias Sociales. Mención Gerencia. E-mail: wileidy_am@yahoo.com. Código Postal 4002. 


\title{
A Systemic Vision of the Rise of Popular, Alternative and Community Radio Stations in Zulia
}

\begin{abstract}
The objective of this study centered on establishing the subsystems for popular, alternative and community radio stations (PAC) in the State of Zulia. The event was approached from the systems theory of administration, describing the elements that make up each subsystem. Research is of the descriptive, trans-sectional type. The population consisted of 25 PAC radios, set up by the National Telecommunications Commission in the Zulia region by applying a population census. Data was collected using interviews through a semi-structured questionnaire. Among the results, elements appeared such as: technical, political and social interconnectivity as well as a sense of ownership and identification with the communications work. Conclusions were that the system under which the radios are developing is made up of five subsystems: objectives and values, structural, psychosocial, technical and administrative. Integration of these five subsystems make up the system.
\end{abstract}

Key words: Popular, alternative and community radios, subsystems, structures, relations.

\section{Introducción}

Desde la década de los ochenta en el estado Zulia se inicia el reconocimiento de las radios populares, alternativas y comunitarias como instrumentos de expresión de la población; orientadas al bienestar social y a la transformación de una comunicación basada en la acción social de los individuos que desean expresarse de manera democrática.

El auge que han tenido, especialmente por el apoyo gubernamental ha propiciado la conformación de un marco legal amparado por la Constitución de la República Bolivariana de Venezuela (Asamblea Nacional Constituyente, 1999), Ley Orgánica de Telecomunicaciones (Asamblea Nacional Constituyente, 2000), y el Reglamento de Radiodifusión Sonora y Televisión Abierta Comunitaria de Servicio Público, sin fines de lucro (Asamblea Nacional Constituyente,
2002); entre otras, formalizando así la comunicación popular, alternativa y comunitaria en Venezuela.

Bajo un enfoque pragmático, el rápido crecimiento de las radios populares, alternativas y comunitarias en el Zulia ha generado el desarrollo de una estructura informal, carente de coherencia, sistemátización, organización, lo que ha conllevado a la dispersión en los objetivos y formalidades para los cuales han sido creados.

Con base en lo anterior y en la teoría de sistemas se identifican los elementos que conforman los subsistemas de las radios en el estado Zulia, y comprender la estructura y funcionamiento del sistema de las radios antes mencionadas. El evento fue abordado desde la teoría de sistemas en la administración, de acuerdo a Munch (2005:46) se entiende que:

"la organización como un sistema de multitudinarias actividades y relaciones; desarrolla un esquema para la des- 
Visión sistémica del surgimiento de las Radios Populares, Alternativas y Comunitarias...

Useche, María Cristina y Artigas, Wileidys

cripción del mundo empírico del que se ocupa la administración; la empresa es un sistema hecho por el hombre; sus partes internas trabajan juntas para alcanzar objetivos establecidos, y sus partes externas trabajan para lograr la interacción con el ambiente".

Según los precursores, a saber, March y Simon, (1980) y Kast y Rosenzweig (1994) para comprender que las partes separadas no podrán explicar el todo, ya que son las interacciones las que determinan el funcionamiento del todo, y para ello es imprescindible conformar los elementos que se encuentran interrelacionados y poder construir las partes en las que se dividen los sistemas $y$ de las cuales depende el funcionamiento.

Razón por la cual el tipo de investigación es descriptiva-transeccional. La técnica aplicada en la recolección de datos fue la entrevista, obteniéndose éstos de manera verbal por parte de los sujetos informantes. Dicha técnica requirió de un instrumento, semi-estructurado ya que genera un proceso de interacción social que permite descubrir la realidad estudiada y ampliar el contenido de aquello que considere relevante para la investigación. Asimismo, se hizo una revisión documental sobre aquellas recopilaciones de información que se pueden tomar en cuenta para fines cognoscitivos; a saber, textos especializados, leyes y reglamentos, entre otros, que inciden en las radios populares, alternativas y comunitarias. La población estuvo conformada por todas las radios populares, alternativas y comunitarias que se desempeñan en el estado Zulia habilitadas por la Comisión Nacional de Telecomunicaciones (CONATEL) que ascienden a 25 , por lo que se implementó un censo poblacional; siendo los agentes de información representantes de cada radio.

Sin embargo, es importante resaltar que de las 25 radios sólo fueron ubicadas 21 , debido a que 3 de ellas se encuentran con problemas técnicos y actualmente no están funcionando; y 1 de ellas no pudo ser ubicada con los datos que se encuentran en CONATEL.

\section{La Radio en Latinoamérica y su incidencia en Venezuela}

Sobre el surgimiento de la radio como medio de comunicación López (2006) señala que la necesidad de comunicación del hombre lo llevó a la búsqueda de alternativas de medios masivos. Sin embargo, en Latinoamérica el avance se ha relacionado a los progresos tecnológicos externos, mediante un proceso de redimensionamiento de los medios hacia el ámbito comunitario, especialmente al acceso a personas de forma individual y/o colectiva que permitan conocer y entender puntos de vista sobre diferentes tópicos.

Intentar superar barreras locales para difundir y promover la información impulsó la creación de una serie de medios de comunicación que fuesen herramientas para ampliar el acceso a la información. Con el transcurrir del tiempo los medios de comunicación se han convertido en entes constructores de la realidad local, regional, nacional e internacional, con toda la implicancia social, política y cultural, conllevándola a desempeñar un rol preponderante a nivel socio-educativo en las sociedades (Colussi, 2006). 
En tal sentido, organismos con gran alcance internacional como la Organización de las Naciones Unidas (ONU) (1948:45) ha fijado metas referente a la comunicación; a saber; garantía de la libertad de opinión, de expresión y de la libre corriente de la información. En el Artículo 19 de la Declaración Universal de los Derechos del Hombre se expresa la:

“...libertad de opinión y de expresión; este derecho incluye el de no ser molestado a causa de sus opiniones, el de investigar y recibir informaciones y opiniones y el de difundirlas, sin limitación de fronteras, por cualquier medio de expresión".

En el ámbito comunicacional, la radio constituye un medio importante, tanto por la información que transmite como por la relación impersonal entre emisor y receptor; asimismo, su bajo costo permite que la colectividad tenga mayor acceso a ella. Al respecto MacBride y col. (1993), obtuvieron en su investigación que para 1950, cerca de cincuenta países no tenían instalaciones de radiodifusión, pero esta cifra se había reducido a tres en 1973. Ello gracias a que la capacidad de transmisión que puede ser una oferta discursiva en vivo, en interacción con lo grabado, generada por un centro emisor, con una variada información, entrelazada con diferentes tipos discursivos, que se puede recibir realizando otras actividades sociales que requieran visión y atención.

En América Latina, las estaciones operadas por agencias gubernamentales coexisten con la radiodifusión religiosa y educativa y con las redes comerciales. Tradicionalmente, el financiamiento de los servicios de radiodifusión se ha realizado mediante subsidios estatales, hono- rarios de licencias o gastos de publicidad. Pero la elevación de los costos obligó a la mayoría de los sistemas a diversificar sus fuentes (MacBride y col., 1993), usando como estrategia la comercialización de espacios publicitarios para la obtención de suficientes ingresos para el activo funcionamiento de las mismas.

Ello mermó el origen y la razón de ser de las radios: una comunicación más abundante entre los pueblos, mejor y libre, con un enfoque democrático para que pueda escucharse las voces y convertirse en actores activos del proceso de comunicación, aumentar la diversidad de los mensajes, y mejorar la participación de las personas. Esta necesidad de participación ha surgido porque la información y formación fueron suplantadas por el entretenimiento y la persuasión comercial.

La orientación de los medios se ha convertido en preocupación desde finales de la década de los cuarenta, en Colombia comienzan a usar sus espacios radiofónicos para proveer educación en beneficio de la población pobre del área rural, a partir de la cual se fue generando un movimiento en América Latina con la finalidad de defender un proyecto de radio que garantice el acceso a la palabra a todos los grupos o sectores de la población a quienes siempre se ha negado su uso; tomar en cuenta las necesidades prioritarias de las comunidades a las que sirven; representar la diversidad cultural del entorno; apoyar y promover los cambios sociales necesarios para lograr una sociedad más justa, entre otros (Geerst y Van Oeyen, 2001).

Surge entonces, la necesidad de otro tipo de medios comunicativos: Ios llamados medios alternativos. Es decir: me- 
Visión sistémica del surgimiento de las Radios Populares, Alternativas y Comunitarias...

Useche, María Cristina y Artigas, Wileidys

dios de comunicación no centrados en la dinámica empresarial, tampoco en el espectáculo de la vida sino en la vida misma, en la lucha de la vida. Esta gama de medios se reconocen como alternativos y tiene como objetivo convertirse en un instrumento popular, una herramienta en manos de los pueblos para servir a sus intereses (Colussi, 2006). En este orden de ideas, según Astudillo y Pino (2007) la historia de la comunicación comunitaria, popular y alternativa en Latinoamérica ha vivido diversos procesos de fortalecimiento, tejiéndose los procesos desde diversos escenarios, luchas de la comunicación y por ella.

La radio popular surge a partir de dos vertientes: una católica que emerge de las experiencias de la radio educativa y otra sindical, que tiene su origen en las emisoras mineras en Bolivia entre los años 50 y 60 . Ambas iniciativas respondieron al permanente y creciente clamor latinoamericano por la justicia y la paz; por un lado inspirada en la liberación y pedagogía de Freire que conjugadas hacen que a fines de los 70's se acuñe el término de radio popular.

El grado de vigencia e incidencia de los medios populares tiene que ver con particularidades de los pueblos donde se desarrolla. De acuerdo a Bisbal (2007) este surgimiento de los medios, especialmente en los países menos desarrollados que han estado relacionados a los cambios y tendencias políticas que los países han experimentado cambios coyunturales y estructurales. Así, a mediados de los 80 's las primeras radios comunitarias comenzaron a surgir en un escenario de apertura democrática en los dos países más sacudidos por la dictadu- ra militar, como fueron Argentina y Chile. De acuerdo a Geerst y Van Oeyen (2001:56).

En Argentina el auge de las 2500 radioemisoras fue realmente un fenómeno histórico. A partir de los años 90 , las radios comunitarias comienzan a proliferar en toda América Latina. De los 12 países investigados solamente en la República Dominicana no se hace mención de ese tipo de emisoras.

Mientras que Venezuela, Bolivia, Ecuador, Perú y El Salvador se toman en cuenta como países de alto desarrollo de las radios comunitarias con existencia de coordinaciones en el ámbito nacional. Destacando que la radio debe constituirse como un espacio democrático y participativo, donde los sujetos excluidos puedan colaborar en la creación de formas nuevas y contenidos innovadores de acuerdo con el mismo proceso de cambio social.

En este sentido, se observa como los medios populares, alternativos y comunitarios fueron surgiendo en Latinoamérica en función de establecer una forma de comunicación alterna con respecto a movimientos o luchas de carácter social, y que a través del tiempo se fueron consolidando relaciones y procesos para la legitimación de las mismas.

\section{Impulso de las Radios Populares, Alternativas y Comunitarias en Venezuela.}

En ese contexto comunicacional latinoamericano, Venezuela establece las diferencias entre medios públicos y privados, señalando que para 1999 el parque público de medios apenas contaba con la señal de Venezolana de Televisión (VTV), 
una señal radial en amplitud modulada (630 AM) para el centro del país y una señal en frecuencia modulada (91.1 FM). Sin embargo el panorama cambió drásticamente, no sólo en cantidad de medios de propiedad directa del Estado, sino en el volumen de medios que llamaremos "para-estadales" o "para-públicos" ya que nacen por iniciativa del gobierno quien los incentiva jurídicamente, publicitariamente y además los ayuda en la compra de los equipos requeridos para su instalación e inmediata puesta en producción (Bisbal, 2007).

Las emisoras comunitarias (también llamadas locales o alternativas, y, últimamente, planteadas como radios ciudadanas) generan una ruptura en cuanto a los parámetros establecidos que evidenciaban el carácter funcionalista y técnico del medio. La nueva forma de utilización del medio hace que estas emisoras no puedan ser definidas por la cobertura (mayor o menor potencia), ni por las características técnicas (AM o $\mathrm{FM}$ ), ni por sus propietarios, y mucho menos por la programación que éstas puedan tener. Lo esencial era su carácter comunitario, los objetivos sociales o por el proyecto comunicativo que requerían consolidar (Astudillo y Pino, 2007).

En Venezuela, a finales de $2002 \mathrm{e}$ inicios de 2003, las radios ciudadanas comienzan a proyectar principios fundamentales de la ciudadanía: libertad, igualdad y fraternidad. Derechos y deberes compartidos que, desde la radio, deben defenderse. La expansión de los medios alternativos y comunitarios audiovisuales en Venezuela se inicia posterior a la promulgación de la Ley de Telecomunicaciones, en la cual se prevé su posibilidad de habilitación en el espectro radioeléctrico (Raydan, 2009).

Ante tal posibilidad el movimiento de medios comunitarios (más de 200 colectivos a nivel nacional) luchan por su autonomía y consolidación junto a las organizaciones de base, para impulsar estos medios como canal formativo con propuestas integrales para la dinamización cultural de las comunidades, generando una posibilidad real del ejercicio de la comunicación y la construcción de mensajes en todas sus etapas, en pro de la democratización comunicacional y el acceso directo de todos a la tecnología y administración del medio (Florido y col., 2007).

En Venezuela se pueden diferenciar tres grupos de medios, dos de ellos de tradicional vigencia y el tercero -lo alternativo- que está adquiriendo cada vez mayor importancia social. El primer grupo, está constituido por los medios privados, a menudo llamados "independientes", cuyo objetivo originalmente fue el lucro. El segundo grupo, incluye a los medios que son voceros de determinada tendencia ideológica, política, religiosa; a este grupo pertenecen también los medios que son propiedad del Estado y que tradicionalmente han sido voceros de los gobiernos. El tercer grupo, lo conforman los medios alternativos, entre los cuales se incluye a los medios comunitarios, que surgen como expresión de sectores sociales excluidos de la oferta tradicional (Dragnic, 2007).

Los medios del tercer sector se conocen como Populares, Alternativos y Comunitarios (nombrados en conjunto o de forma separada). Para la Asociación Latinoamericana de Escuelas Radiofónicas (ALER) (2001) el carácter popular 
Visión sistémica del surgimiento de las Radios Populares, Alternativas y Comunitarias...

Useche, María Cristina y Artigas, Wileidys

hace referencia a poseer alta audiencia, promover el protagonismo, logro de la identificación con los sectores populares; así como, la contribución al fortalecimiento de las prácticas culturales, productivas, sociales y políticas. Por su lado, lo alternativo se refiere a la consolidación de un proyecto de radio garantizando el acceso a la palabra a todos, tomando en cuenta las necesidades prioritarias de las comunidades, representando la diversidad cultural del interno, sin dejar de lado la promoción de los cambios sociales necesarios.

$Y$ con respecto al carácter comunitario se centra en la contribución al desarrollo por medio de la democracia, defendiendo los derechos humanos y la diversidad cultural en diversos ámbitos: local, regional y nacional. Correlacionando esos tres aspectos se puede aseverar que el carácter popular, alternativo y comunitario concibe que la comunidad tenga una garantía de acceso a la información, se trabaje en abordar y superar necesidades e intereses de cada comunidad y se fortalezca de la diversidad cultural autóctona de cada ámbito.

Desde la configuración de las radios populares, alternativas y comunitarias (PAC), el gobierno revolucionario de Venezuela ha apoyado legal, política y gubernamentalmente a las mismas, mediante el establecimiento de políticas de desarrollo comunitario y participación protagónica del pueblo mediante estas herramientas comunicacionales, coadyuvando en la instalación, operación y generación de contenidos audiovisuales o radiales a través de los llamados Servicios de Radiodifusión Sonora y televisión Abierta Comunitaria sin Fines de Lucro.
A pesar de los avances, el crecimiento sigue con la irrupción de la radio en frecuencia modulada (FM) que en el país se instala hacia finales de la década de los ochenta y que marca el inicio de la importancia de la radio en el país (Bisbal, 2007). De esta forma, entre 1984 y 1999 se entregaron 70 concesiones de radio AM mientras que las FM alcanzaban las 236, más de un $300 \%$ que las AM, reflejando la primacía de los grupos privados sobre los públicos (Radio Mundial, 2009).

En síntesis, las radios populares, alternativas y comunitarias han tenido un desarrollo diferenciado en Venezuela, utilizándose como medio de protesta y expresión del pueblo, en contraposición de la desviación de los fines de las radios comerciales, conjugando elementos autóctonos de popularidad y comunidad local, regional y nacional.

\section{Surgimiento de las Radios Populares, Alternativas y Comunitarias en el estado Zulia}

Entre los años 1983 y 1984 se creó la primera radio popular, alternativa y comunitaria en la región zuliana, denominada TROPICAL, la cual nace acompañada de una señal de televisión abierta identificándose como Televisora Vecinal Canal 7 y saliendo como Tropical en el díal 105.5; sin embargo, la habilitación no fue obtenida hasta el 2003, la radio fue habilitada por 25 años y obtuvo la concesión por 5 años (Vergara, 2010).

La habilitación, de acuerdo a la Ley Orgánica de Telecomunicaciones (Asamblea Nacional Constituyente, 2000) significa que legalmente está autorizada para 
funcionar, mientras que la concesión hace referencia al permiso a ocupar determinado dial, ésta es la que se renueva constantemente; sin embargo hasta el momento no se ha renovado ninguna concesión a las radios comunitarias.

En el Cuadro 1 se puede observar cronológicamente el surgimiento de las emisoras de radios desde el año 1983 hasta el año 2008. La segunda radio, en orden cronológico es Luz para Todos, que se creó en 1990, según Villalobos (2010a) motivado por razones personales, el director actual de la fundación fue el creador, es técnico en electrónica y computación y construyó artesanalmente el transmisor a través del cual salían al aire.

Sin embargo, en ese año como las radios comunitarias aún no se encontraban establecidas en ninguna ley o reglamento era cotidiano el cierre de las mismas, especialmente porque la tendencia que se mostraba era hacia el cristianismo

\section{Cuadro 1 \\ Creación/habilitación de las Radios Populares, Alternativas y Comunitarias en el estado Zulia}

\begin{tabular}{lcl}
\hline Año de creación & Habilitación & Radio \\
\hline $1983 / 1984$ & 2003 & Tropical \\
1990 & 2007 & Luz para todos \\
1998 & 2003 & Perijanera \\
$1998 / 1999$ & 2003 & Máxima \\
2000 & 2003 & Amistad (única renovada) \\
& 2003 & La voz del pescador \\
& 2004 & Parroquiana \\
2001 & 2004 & San Rafael \\
& 2003 & Soberana \\
2002 & 2004 & La voz del pueblo \\
2003 & 2007 & Coquivacoa \\
& 2007 & La voz del monte \\
& 2003 & Acción (Inicio en 2005) \\
2007 & 2005 & Curva estéreo \\
No se obtuvo información & 2007 & Voces de Gibraltar \\
& 2008 & Antorcha \\
& 2008 & Samide Estéreo \\
& 2004 & Vereda Libre \\
& 2008 & Bari Karañakae'k \\
& 2008 & Wanüiki \\
& 2006 & Sucrense \\
\hline
\end{tabular}

Fuente: Elaboración propia. 
Visión sistémica del surgimiento de las Radios Populares, Alternativas y Comunitarias...

Useche, María Cristina y Artigas, Wileidys

y la iglesia católica tenía mucho poder ante las autoridades.

Al respecto señala también Villalobos (2010) que hubo períodos intermitentes en los cuales la radio funcionaba o se apagaba, y fue con la reforma constitucional que se comenzó a tener espacios. En la década de los noventa e inicios del 2000 era difícil obtener los permisos; y fue en el 2007 que se logró la habilitación y desde ese entonces se asignó el dial 92.1; dejando claro que en ese dial ya había una emisora de alta potencia Unión Estéreo, que es comercial y no está habilitada. Asegura tener 50 vatios contra 5000 , lo cual deja prácticamente imposibilitados y sin cubrir siquiera el espacio para aquel que sí está permisado.

Seguidamente se encuentra en 1998 la creación de Perijanera, que obtuvo la habilitación en 2003, para el inicio de la misma se integraron pocas personas $y$ luego se fue incorporando a la comunidad y las organizaciones sociales que han ido evolucionando; actualmente en Machiques (López, 2010).

Entre el 1998 y 1999 fue creada Máxima, la cual nace de acuerdo a Yépez (2010) por el interés de periodistas que en el ejercicio profesional vislumbraban una idea de comunicación distinta pero sin ningún basamento legal específico, con 150 vatios y un transmisor artesanal; luego de la constitución se inician las reformas, la Ley Orgánica de Telecomunicaciones (Asamblea Nacional Constituyente, 2000) y el Reglamento de Radiodifusión Sonora y Televisión Abierta Comunitaria de servicio público, sin fines de lucro (2002), iniciando las habilitaciones para ese año y obteniéndose la misma en el 2003, conjuntamente con 30 medios comunitarios en el territorio nacional.

En el año 2000 por su parte fueron creadas 4 radios, a saber: Amistad, La Voz del Pescador, Parroquiana y San Rafael; en el caso de Amistad según Colina (2010) nace en el año 2000 logrando promover el proceso de cambio que ha experimentado el país en los últimos años; señalando que ya han sido renovados; en el caso de la Voz del Pescador, ésta ha estado involucrada en la creación del reglamento que se encuentra vigente. Por su parte, Parroquiana según León (2010) ha venido haciendo un trabajo comunitario y destaca que el avance del medio ha sido difícil en función de la deserción de gente que trabaja en el proyecto y las diferencias políticas que existen en el municipio en que se encuentra.

En el caso de San Rafael, contó con la aceptación no sólo del municipio Mara, sino también de Páez y Padilla, resaltando el apoyo que han obtenido del alcalde de su Municipio y la lucha en la mejora de las condiciones de la sociedad y en la búsqueda de gente que quiera colaborar sin ningún interés adicional (Ocando, 2010).

Para el año 2001 surgen Soberana, la Voz del Pueblo y Coquivacoa; llevando a cabo un trabajo con las comunidades que no siempre ha sido fácil, ya que las limitaciones económicas han hecho que el trabajo sea reducido. Para González (2010a) las comunidades aún no han despertado, no saben el valor de las radios comunitarias con las que cuentan, sobretodo porque las relacionan con una tendencia política y en las comunidades existe la diversidad y todos deben estar relacionados para el bien común independientemente de las preferencias. 
Para el 2002 se creó la Voz del Monte, Samide y Pueblo Estéreo, y según Belloso (2010) la primera inicia el funcionamiento a raíz de las situaciones políticas que se enfrentan en los años 2002 y 2003 , cuando se arma un equipo de gente aclarando la situación de Petróleos de Venezuela (PDVSA) en ese entonces. Se hacían reuniones y se transmitían por otras radios comunitarias.

En el 2003 se crean Acción, Curva Estéreo, Voces de Gibraltar y Antorcha, destacando que las radios que surgen entre el 2002 y 2003 comparten la necesidad de funcionamiento ante las situaciones políticas que enfrentaba el país en ese momento. Para Moreno (2010) el trabajo ha sido arduo, dada las expectativas generadas; así mismos, al momento de funcionar se observan las limitaciones e interferencias en el trabajo, afectando la eficiencia y generando problemas para integrar las comunidades; sin embargo, se ha logrado incrementar la participación en las actividades desarrolladas a través de la radio promoviendo la participación sana, trabajando con toda la gente sin diferencia de credo raza o religión.

En el 2004 nace Vereda Libre, según Cepeda (2010) el funcionamiento es bastante limitado pero han logrado crear una página de Internet para extender su capacidad radial y llegar a más lugares. En el 2006 surgió Bari Karañakae'K, según Abi (2010) fue habilitada para el año 2008 y ha sido de gran ayuda para la comunidad donde se encuentra, ya que están en la montaña y es una de las formas de comunicarse.

En el 2007 germina Wanüiki, que venía realizando un trabajo comunitario desde los años 70 , obtuvo la habilitación en el 2008, convenciendo a la población, ya que no creían en el proyecto y luego de verlo consolidado es que han venido integrándose en el mismo.

Finalizando con la radio Sucrense, Díaz (2010) expresa que aunque obtuvieron la habilitación en 2006 no han logrado salir al aire por no contar con los recursos necesarios, sin embargo no especificó el año de creación ni el de habilitación, sólo reflejó que siguen trabajando para lograr el funcionamiento.

Cabe señalar que, en la conformación de las radios populares, alternativas y comunitarias en el estado Zulia se demarca un trabajo sistémico, al evidenciar elementos, como: la ínter conectividad técnica, política y social; así como, incertidumbre ante la falta de conocimiento legal y de formación técnica especializada, entre otras. De igual forma se detecta un cambio en la concepción comunicacional participativa, ya que se democratiza la información, se impulsan espacios de discusión comunitaria y debate que generan interacciones, donde las personas que viven en las parroquias son los protagonistas y constructores de la comunicación.

El surgimiento de estas radios concibe una comunicación alternativa a las históricas y comunitaria al expresar la práctica social, son instrumentos comunicacionales que facilitan la divulgación del acontecer comunal, opinión ante diversas coyunturas y situaciones que afecten el devenir regional y nacional, así como la contrastación de ideas en un marco de respeto y democracia. De igual forma son los medios que coadyuvan a las reivindicaciones tradicionales y populares, es decir, de la idiosincrasia de las comunidades. 
Visión sistémica del surgimiento de las Radios Populares, Alternativas y Comunitarias...

Useche, María Cristina y Artigas, Wileidys

Asimismo, la comunicación que gestionan estas radios ha estado centrada tanto en el ámbito geográfico como en la creación de mecanismos de interacción con otras comunidades. Esa expresión radial es producto de quienes la elaboran, una manera de reconocer que están allí para promover, debatir ideas, generar espacios de debate y articularse con las esferas sociales en pro de la formación de un medio plural autogestionario.

\section{Una Visión Sistémica del Surgimiento de las Radios Populares, Alternativas y Comunitarias en el estado Zulia}

Visualizado un panorama general de las radios populares, alternativas y comunitarias en el estado Zulia, es preciso llevar a cabo una síntesis de los elementos sistémicos presentes en las mismas, que se pueden observar en la Figura 1. En este sentido, se detectaron 5 subsistemas a saber: el subsistema de objetivos y valores, el subsistema estructural, el subsistema psicosocial, el subsistema técnico y el subsistema administrativo.

Para Kast y Rosenzweig (1994:19) el subsistema de objetivos y valores es "una premisa básica que la organización como subsistema de la sociedad debe lograr (...) por el sistema que la envuelve". Al respecto, se detectó entre los miembros de las comunidades que entre sus objetivos se encuentra informar sobre las necesidades de cada comunidad y las luchas colectivas para mejorar los espacios comunes con equidad, resaltando los elementos axiológicos de las radios y su funcionamiento en el ámbito comunitario.
Figura 1

Subsistemas de las Radios

Populares, Alternativas y Comunitarias en el Zulia

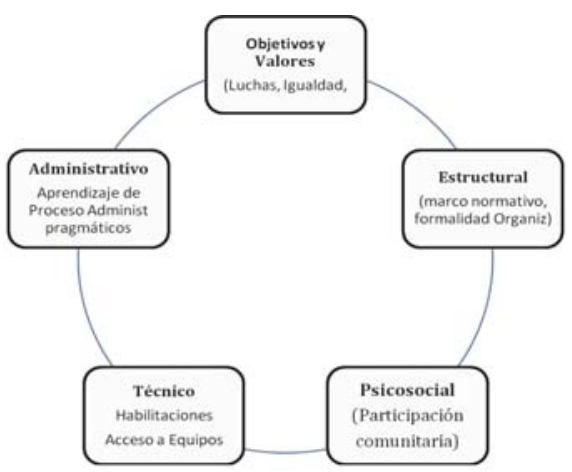

Fuente: Elaboración propia.

Mientras que el subsistema estructural para Kast y Rosenzweig (1994) se refiere a la estructura formal de la organización, como: estatutos, puestos y descripciones de labor de la organización, así como en los manuales de procedimiento. La estructura informal está determinada por interacciones informales entre los participantes en la organización y está estrechamente asociada con el sistema psicosocial.

Al respecto, el mismo lo conforma la creación de la fundación comunitaria, constituidas de conformidad con las formalidades previstas en el Código Civil venezolano y las especificaciones determinadas, deben tener un carácter comunitario y, por ende, podrán ser titulares de la concesión de radiodifusión y de las habilitaciones de radiodifusión sonora abierta comunitarias de servicio público, sin fines de lucro en pro de la comunicación libre y plural de los miembros de las comunida- 
des. La Comisión Nacional de Telecomunicaciones determina el modelo para la constitución de las fundaciones comunitarias en el que, a través de la fijación de las características organizativas, de funcionamiento y de control de tales fundaciones, establece de manera uniforme la forma en que los interesados deberán cumplir con los requisitos establecidos.

El subsistema psicosocial, según March y Simon (1980) es importante al momento de establecer una organización y en lo sucesivo, ya que en éste se organiza en forma adecuada el grupo de trabajo para conocer el comportamiento de los mismos y los eventos que se producen por la interrelación grupal. En las radios este subsistema ha sido el más informal de todos, por no estar normado, no está debidamente establecido, por lo que no existen parámetros de trabajo voluntario. Las personas que participan han sido voluntarias, en muchos casos miembros de organizaciones sociales en las cuales se observa mayor cohesión; sin embargo, ha existido mucha deserción por diferencias políticas o compromisos laborales.

Ello evidencia una incipiente participación comunitaria, que requiere a través de este medio persuadir y estimular a las personas a integrarse e intervenir activamente en las problemáticas comunales. Aunque no debe dejarse de lado que, las personas que permanecen han realizado un trabajo comunitario continuo y en muchos casos no han podido realizar el cambio de directiva en función de la falta de compromiso de otros miembros de la comunidad.

El subsistema técnico de acuerdo a Kast y Rosenzweig (1994) está con- formado por los conocimientos, técnicas, instalaciones y equipos, es decir, requerimientos de trabajo de la organización y la forma de especialización de aptitudes y conocimientos requeridos; los tipos de maquinaria y equipos utilizados, los requerimientos de procesamiento de la información y la disposición que necesita la organización para el desempeño de sus actividades.

En las radios se manejan parámetros de operación, como las habilitaciones, que en la mayoría de los casos se ha obtenido después del funcionamiento, con algunas excepciones. El dial asignado por los organismos ha afectado en 2 casos el funcionamiento de las radios por interferencias. Para ampliar la acción se ha usado internet como herramienta alterna.

De igual forma este subsistema lo conforma la programación, los períodos de transmisión, la banda de operación de las Radios, entre otros; ésta última sólo es otorgada en concesión de uso y explotación por el Ministro de Infraestructura (delegada actualmente a la Comisión Nacional de Telecomunicaciones (CONATEL), la cual está comprendida en la porción del espectro radioeléctrico atribuida al servicio de radiodifusión sonora comunitaria en el Cuadro Nacional de Atribución de Bandas de Frecuencias (CUBANAF) (Asamblea Nacional Constituyente, (2002).Los parámetros técnicos de operación, dejando claro los kilohertz, el ancho de banda y la modulación, que en este caso en Frecuencia Modulada (FM), siendo CONATEL el ente que tiene la potestad de cualquier aprobación de modificación de las características técnicas y la ubicación de los equipos. 
Visión sistémica del surgimiento de las Radios Populares, Alternativas y Comunitarias...

Useche, María Cristina y Artigas, Wileidys

En relación al subsistema administrativo, se encuentra relacionado fundamentalmente con la toma de decisiones para planear y controlar los esfuerzos organizacionales (Kast y Rosenzweig, 1994).

En las radios explícitamente no se establecen lineamientos sobre el mismo, sin embargo, el Reglamento de Radiodifusión Sonora y Televisión Abierta Comunitaria de Servicio Público, sin fines de lucro (Asamblea Nacional Constituyente, 2002), se establece claramente que en el artículo 19 que el patrimonio de las fundaciones debe provenir o estar constituido por aportes, donaciones, o subvenciones, podrá provenir de miembros de la comunidad donde se preste el servicio de radiodifusión sonora comunitaria o el servicio de televisión abierta comunitaria o de otras personas pero nunca de operadores de radiodifusión sonora o televisión abierta. El resto de los procedimientos administrativos son establecidos en la práctica cotidiana, sin embargo se debe aclarar que las normas terminan flexibilizándose en función del valor otorgado al trabajo comunitario.

Asimismo, el artículo 20 del mismo reglamento destaca que los recursos serán destinados a garantizar el funcionamiento y mantenimiento de las redes de telecomunicaciones, la continuidad en la prestación del servicio de que se trate y la realización del objeto para el cual fue constituida, siendo las autoridades u órganos de dirección, administración y control, deberán tener su domicilio o residir en la localidad donde se preste el servicio de radiodifusión sonora comunitaria.

En este sentido, se puede observar vacíos importantes que influyen significa- tivamente en el desempeño de las radios, entre los cuales se pueden mencionar: tiempo de pautas publicitarias en pro de la sostenibilidad económica y comunitaria; así como, lineamientos que permitan el manejo de las radios a través de criterios de autosostenibilidad, sin embargo, éstos pueden ser perfectamente subsanados por parte de la comunidades y aprovechar para generar una propuesta que términos de complementariedad subsane lo indicado, así como estudiar la viabilidad de introducirlos en una posible actualización del reglamento existente.

\section{Conclusiones}

Los subsistemas que han conformado las radios PAC se centra en la interacción social; por lo que, el sistema comunicacional de estos medios de comunicación se ha basado en el sentido de pertenencia, la identidad y la participación de las comunidades en el trabajo de las radios PAC, permitiendo que se integren los subsistemas bajo los cuales se desenvuelven, para que todas, como un todo, desarrollen sus objetivos, actualicen información con y sobre el entorno, en pro de un equilibrio sistémico comunal.

La comunidad ha encontrado en las radios $\mathrm{PAC}$ un medio de expresión social-local, que no sólo facilita la comunicación, sino que enseña a la misma diversas formas de interacción, promoción y difusión de luchas, de problemas, pero también de unión y cooperación.

Resulta importante destacar que, en las radios PAC la delimitación de un sistema no sólo está basado en la extensión geográfica, física, u organizacional, 
sino también en todo el acontecer que directa o indirectamente incide en la comunidad. Ello plantea la necesidad de abarcar lo que se encuentre dentro de estos límites, y observar qué hay un poco más allá. Esto delimita la extensión de la ética del sistema, y el impacto que puede generar, por lo que, se involucra más y mejor con el medio en que se desenvuelven.

En términos de complementariedad, sería interesante indagar sobre la equifinalidad del sistema para tener una comprensión estratégica sobre los diversos procedimientos y acciones empleadas, para alcanzar los fines, lo que le imprimirá la diferencia en su entorno, así como cotejar criterios e intereses de cada emisora radial.

\section{Referencias bibliográficas}

Asamblea General de las Naciones Unidas (2010). Declaración Universal de los Derechos Humanos. 1948. Extraído de http://www.pfcmc.com/es/documents/udhr/ en fecha: Mayo 4, 2010.

Asamblea Nacional Constituyente (1999). Constitución de la República Bolivariana de Venezuela. Gaceta Oficial Extraordinaria No. 5453 el 24 de Marzo de 2000. Venezuela.

Asamblea Nacional Constituyente (2000). Ley Orgánica de Telecomunicaciones. Publicada en gaceta oficial $N^{\circ} 36970$ de la República Bolivariana de Venezuela.

Asamblea Nacional Constituyente (2002). Reglamento de Radiodifusión Sonora y Televisión Abierta Comunitaria de servicio público, sin fines de lucro. Decreto N01.521 de fecha 08/01/2002.

Asociación Latinoamericana de Escuelas Radiofónicas (ALER). (2009).Un camino por recorrer. Proyecto Político Comunicati- vo de la Radio Popular Latinoamericana. Apta para comentar, enriquecer, mejorar, ampliar. Proceso preparatorio. XIII Asamblea General de ALER. [Versión electrónica] Publicación web extraída de www.aler.org.ec en fecha: 15/11/2009.

Astudillo, A y Pino, J. (2007). Radio local, opinión pública y participación ciudadana. [Versión electrónica]. Extraído de http://www.scielo.org.co/scielo.php? Pid $=$ S0120-8232008000100007\& script=sci_arttext.

Bisbal, M. (2007). Los Medios en Venezuela ¿Dónde Estamos? Extraído de http://redalyc.uaemex.mx/redalyc/ src/inicio/ArtPdfRed.jsp?iCve= 12216401, en fecha 13/10/09.

Colussi, M. (2006). Medios de comunicación alternativos: Una guerra popular. [Versión electrónica] http://www.aporrea.org/medios/ a21188.html en fecha $28 / 09 / 09$.

Dragnic, O. (2007). Los medios de comunicación social en el socialismo del siglo XXI. [Versión electrónica] http://www. scielo.org.ve/scielo. .php?pid=S1315$64112007000200011 \&$ script $=$ sci arttext en fecha, 10/10/09.

Florido, R.; Peraza, W.; Cortez, A. y otros (2007). Las Radios Comunitarias Presente y Futuro en la Construcción del Socialismo del Siglo XXI. Documento no publicado. Barquisimeto, Venezuela.

Geerts, A. y Van Oeyen, V. (2001). La radio popular frente al nuevo siglo: estudio de vigencia e incidencia. Asociación Latinoamericana de Escuelas Radiofónicas. Quito, Ecuador. [Versión electrónica] www.aler.org.ec.

Kast, F. y Rosenzweig, J. (1994). Administración en las organizaciones. Enfoque de sistemas y de contingencias (Cuarta edición). México, D.F. Editorial Mc Graw Hill Interamericana de México, S.A. 
Visión sistémica del surgimiento de las Radios Populares, Alternativas y Comunitarias...

Useche, María Cristina y Artigas, Wileidys

López, J. (2006). Manual Urgente para Radialistas apasionadas y apasionados. Tomo I. Ministerio de Comunicación e Información. Caracas, Venezuela.

MacBride, S. y otros (1993). Un solo Mundo Voces Múltiples: Comunicación e Información en nuestro tiempo. Informe de la Comisión Internacional para el estudio de los Problemas de Comunicación. México. Fondo de Cultura Económica.

March, J. y Simon, H. (1980). Teoría de la Organización (4ta edición). Barcelona, España. Editorial Ariel.

Münch, L. (2005). Administración y Estilos de Gestión. La clave de la competitividad. México. Trillas. México D.F.

Organización de las Naciones Unidas (ONU) (1948). Declaración Universal de Derechos Humanos. [Versión Electrónica]. Http://www.un.org/es/documents /udhr/index.shtml, en fecha: 16 de julio de 2010.

Radio Mundial (2009). La historia de la entrega de concesiones en Venezuela, y el nacimiento del latifundio mediático. [Versión electrónica] www.radiomundial.com.ve en fecha: Enero 13, 2009.

Raydan, R. (2009). Democratización de la comunicación se refleja en 443 medios alternativos. [Versión electrónica] http://www.abn.info.ve/noticia.php? artículo $=90655 \& l e e=5$, en fecha $28 /$ $09 / 09$.

\section{Entrevistas}

Abi, Rodrigo. Representante de la Radio Bari Karañakae'k. Maracaibo, Venezuela. 21 de mayo de 2010.

Belloso, Ender. Representante de la Radio La Voz del Monte. Carrasquero, Venezuela. 31 de Mayo de 2010.

Cepeda, M. Representante de la Radio Vereda Libre. Maracaibo, Venezuela. 17 de Mayo de 2010.

Colina, H. Representante de la Radio Amistad. Maracaibo, Venezuela. 12 de mayo de 2010.

Díaz, J. Representante de la Radio Sucrense. Maracaibo, Venezuela. 20 de mayo de 2010.

González, S. Representante de la Radio La voz del Pueblo. Maracaibo, Venezuela. 06 de mayo de 2010.

Leon, E.. Representante de la Radio Parroquiana. Maracaibo, Venezuela. $21 \mathrm{de}$ mayo de 2010.

López, J. Representante de la Radio Perijanera. Maracaibo, Venezuela. 20 de Mayo de 2010.

Moreno, M. Representante de la Radio Acción. Maracaibo, Venezuela. 07 de mayo de 2010.

Ocando, C. Representante de la Radio San Rafael. San Rafael del Moján, Venezuela. 03 de junio de 2010.

Vergara, A. Representante de la Radio Tropical. Maracaibo, Venezuela. 22 de mayo de 2010.

Villalobos, E. Representante de la Radio Luz para Todos. Santa Cruz de Mara, Venezuela. 21 de mayo de 2010.

Yépez, V. Representante de la Radio Máxima. Ciudad Ojeda, Venezuela. 01 de junio de 2010. 